\title{
Globale Landwirtschaft vor alten und neuen Herausforderungen
}

\author{
Die globale Landwirtschaft steht vor enormen Herausforderungen. Um diesen zu begegnen, wurden kürzlich \\ zwei internationale Berichte vorgelegt: das International Assessment of Agricultural Knowledge, Science and \\ Technology for Development und der World Development Report 2008. Landwirtschaft ist zentral für die \\ Bekämpfung der Armut: In dieser Botschaft sind sich die beiden Berichte \\ einig. Bezüglich der Potenziale des Marktes und neuer Technologien \\ gehen die Meinungen jedoch auseinander. Die weitaus meisten \\ landwirtschaftlichen Betriebe sind klein und kapitalschwach - dies \\ darf man bei der Diskussion der globalen Perspektiven nicht vergessen.
}

Markus Giger, Hans Hurni,

Brigitte Portner, Urs Scheidegger

Global Agriculture: Enduring and Emerging Challenges | GAIA 17/3 (2008): 280-286

Keywords: agriculture, equity, IAASTD (International Assessment of Agricultural Knowledge, Science and Technology for Development), natural resources, poverty, sustainable development, World Development Report

\section{Neues Interesse an der Landwirtschaft}

Seit 1960 ist der globale landwirtschaftliche Ertrag um rund zwei Prozent pro Jahr gestiegen und ermöglichte trotz Bevölkerungswachstum eine höhere Kalorienversorgung pro Kopf (Tilman et al. 2002). Diese Zunahme hat vor allem in naturräumlich, technologisch und strukturell begünstigten Gebieten stattgefunden. Länder in Afrika südlich der Sahara sowie kleinbäuerliche Regionen in Lateinamerika und Teilen Asiens konnten hingegen die Flächenproduktivität kaum steigern (FAO 2006). Entsprechend hat zwar der Anteil der Hungernden und Unterernährten seit 1990 abgenommen, aber in den Entwicklungsländern ist die absolute Zahl der Unterernährten im Zeitraum 1995/1997 bis 2001/ 2003 wieder gestiegen (UN 2006). Die aktuelle Nahrungsmittelkrise hat diesen Trend noch verschärft. Landwirtschaftliche Entwicklung ist daher zentral in der Bewältigung von Hunger und Armut, zumal im ländlichen Raum noch immer etwa drei Milli-

Kontakt: Dipl.-Ing. agr. Markus Giger | Universität Bern | Geographisches Institut | Centre for Development and Environment | Hallerstr. 10 | 3012 Bern | Schweiz | Tel.: +41 316313752 | E-Mail: markus.giger@cde.unibe.ch

Prof. Dr. Hans Hurni | Universität Bern | Geographisches Institut | Centre for Development and Environment | Bern | Schweiz | E-Mail: hans.hurni@cde.unibe.ch

MSc Brigitte Portner | Universität Bern | Geographisches Institut | Centre for Development and Environment | Bern | Schweiz |

E-Mail: brigitte.portner@cde.unibe.ch

Prof. Dr. Urs Scheidegger | Schweizerische Hochschule für Landwirtschaft | Zollikofen | Schweiz | E-Mail: urs.scheidegger@shl.bfh.ch arden Menschen leben - knapp die Hälfte der Weltbevölkerung - und drei Viertel aller Armen (Ravallion et al. 2007).

Die internationale Gemeinschaft hat die Landwirtschaft lange vernachlässigt. So flossen 198016 Prozent der Entwicklungshilfe in die Landwirtschaft, 2005 weniger als fünf Prozent (World Bank 2007). Nun wurden im letzten Jahr zwei wichtige internationale Bestandsaufnahmen zur Landwirtschaft vorgelegt, eine von der Weltbank mit dem Weltentwicklungsbericht 2008 (World Development Report 2008: Agriculture for Development) (World Bank 2007), die zweite als Resultat eines zwischenstaatlichen Assessments, das International Assessment of Agricultural Knowledge, Science and Technology for Development (IAASTD 2008a), auch Bericht des Weltagrarrats genannt.

Die Publikationen fallen in eine Zeit, in der die Preise in der Landwirtschaft erstmals seit der Ölkrise von 1973 wieder steigen (FAOSTAT 2008). Neben der relativ neuen Nachfrage nach Biotreibstoffen wächst der Bedarf an landwirtschaftlichen Produkten bereits seit einigen Jahren stark: Die Nachfrage nach Getreide wird von 2000 bis 2050 um 75 Prozent steigen, diejenige nach Fleisch sogar um 100 Prozent (IAASTD 2008b). Zudem ist zwischen 1980 und 2000 die jährliche Zunahme der Getreideerträge gesunken und der internationale Lagerbestand auf wenige Wochen Reserve geschrumpft (FAO 2000a).

\section{Der Kontext: alte und neue Herausforderungen}

\section{Soziale Probleme: Ungleichheiten und Armut}

Niedrige Transportkosten, globale Kommunikationssysteme, international tätige Unternehmen und die Reduktion von Handelsbarrieren haben unterschiedlichste landwirtschaftliche Produk- 
tionssysteme in ein globales System eingebunden und gleichzeitig die Unterschiede zwischen den Systemen akzentuiert (FAO 2005). Durch die Entwicklung der „modernen“ Landwirtschaft, wie sie heute in Industrieländern und in Teilen der Länder des Südens praktiziert wird, erhöhte sich die Arbeitsproduktivität in Europa in den letzten hundert Jahren auf das 25- bis 200-Fache. Konnte vor einem halben Jahrhundert eine landwirtschaftliche Arbeitskraft rund zehn Hektar Land bebauen und jährlich bis zu zehn Tonnen Getreide produzieren, kann sie heute auf 200 Hektar einen Ertrag von etwa 2000 Tonnen im Jahr erzielen (Mazoyer 2001, S. 6). Dieses starke Wachstum basiert in erster Linie auf einer markanten Zunahme des Einsatzes fossiler Energie, einerseits durch die Mechanisierung, andererseits durch den massiven Einsatz von Mineraldüngern und Pestiziden, sowie auf dem Anbau von Hochertragssorten (IAASTD 2008a).

Auch ein Teil der Kleinbauern konnte - unter dem Einfluss der Grünen Revolution - die Erträge steigern, doch die meisten konnten die kapitalintensive Entwicklung nicht mitmachen. Und so steht heute einer riesigen Mehrheit von Kleinstbetrieben eine kleine Minderheit gut ausgerüsteter Großproduzenten gegenüber (Tabelle 1).

Im Gegensatz zu Betrieben im Norden können kleinbäuerliche Betriebe im Süden nicht auf staatliche Unterstützung zählen und leiden daher doppelt unter den Subventionen der reichen Länder. Zudem haben fast alle Entwicklungsländer ländliche Regionen und die Landwirtschaft gegenüber urbanen Gebieten sowie der Industrie und dem Dienstleistungssektor systematisch benachteiligt. Gesellschaftliche und politische Machtverhältnisse machen Korrekturen schwierig und Fortschritte sind nur allmählich erkennbar. In landwirtschaftlich dominierten Ländern, zu denen alle ärmsten Entwicklungsländer gehören, werden nach wie vor nur rund fünf Prozent des Staatsbudgets für Investitionen in die Landwirtschaft verwendet, während Industrieländer mehr als zehn Prozent dafür investieren (Fan et al. 2008).

Disparitäten bestehen nicht nur in der Kapitalausstattung, den Betriebsgrößen, der Arbeitsproduktivität und der Agrarstützung, auch in die landwirtschaftliche Forschung wird im Süden relativ zur Bedeutung des landwirtschaftlichen Sektors weniger investiert als im Norden (Hurni et al. 2001, Pardey und Beintema 2001) (siehe Abbildung). Die landwirtschaftliche Produktivität ist also nicht trotz ausreichender Forschung teilweise niedrig geblieben. Vielmehr ist die geringe Forschungsintensität in vielen Entwicklungsländern mindestens ein Teil der Erklärung.

TABELLE 1: Betriebsgrößen in ausgewählten Weltregionen (von Braun 2005 S. 259).

\begin{tabular}{lc}
\hline Weltregion & durchschnittliche Betriebsgröße (ha) \\
\hline Afrika & 1,6 \\
\hline Asien & 1,6 \\
\hline Lateinamerika und Karibik & 67,0 \\
\hline Westeuropa & 27,0 \\
\hline Nordamerika & 121,0 \\
\hline
\end{tabular}

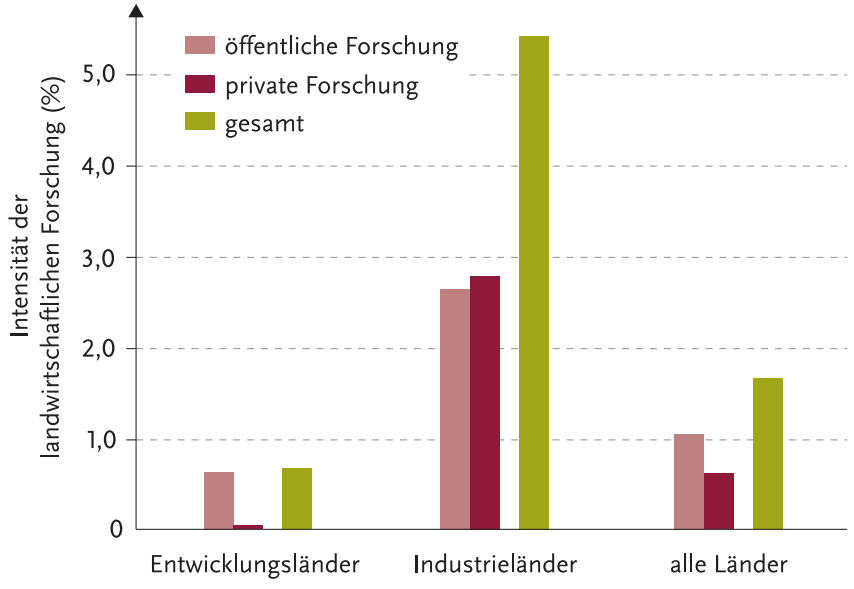

ABBILDUNG: Intensität der privaten und öffentlichen landwirtschaftlichen Forschung von Entwicklungsländern und Industrieländern (nach Pardey und Beintema 2001, S. 14). Landwirtschaftliche Forschungsintensitäten vergleichen die Ausgaben für landwirtschaftliche Forschung mit dem Wert des von der Landwirtschaft erwirtschafteten Bruttosozialprodukts.

Daraus resultieren zwei Fragen: 1. Ist die Landwirtschaft im Süden überhaupt noch eine Grundlage für eine umfassende Entwicklung und Armutslinderung? 2. Wie groß ist der Anteil derjenigen, die in anderen Sektoren und im urbanen Raum ihre Zukunft finden können?

Zu diesen Fragen hat in Entwicklungsagenturen (UN 2007) und akademischen Kreisen eine intensive Diskussion stattgefunden. Konsens ist: Investitionen in die Landwirtschaft sind eines der effizientesten Mittel, um Armut zu verringern (Meinzen-Dick et al. 2004). Über die geeigneten Strategien ist man sich jedoch uneinig, wie die Gegenüberstellung der Berichte zeigen wird.

\section{Umweltprobleme: Landwirtschaft als Verursacherin und Betroffene}

Landwirtschaft basiert auf der Nutzung von Ökosystemen. Deshalb hat die Vervielfachung ihrer Leistungen Auswirkungen auf die Ökosysteme und die Lebensgrundlagen der Menschen. Andererseits ist die Landwirtschaft direkt von den Umweltbedingungen abhängig und kann von Umweltproblemen daher auch negativ betroffen sein:

- Die Umwandlung natürlicher Ökosysteme zur Produktion von Nahrung, Fasern, Energie und Holz ist weltweit eine der wichtigsten Ursachen für den Biodiversitätsverlust (MA 2005, S. 2, IAASTD 2008b).

- Bewässerung und Viehhaltung gehören zu den größten Wasserverbrauchern und -verschmutzern (MA 2005). 15 bis 35 Prozent der Wasserentnahmen für die Landwirtschaft werden als nicht nachhaltig eingestuft (World Bank 2007, S. 182).

- Bodendegradierung betrifft weltweit mehr als die Hälfte der landwirtschaftlich genutzten Böden (Wood et al. 2000, S. 49). Die häufigsten Schäden - Wind- und Wassererosion - werden meist durch unangepasste Bewirtschaftungsmethoden sowohl auf kleinbäuerlichen Betrieben wie auch in mechanisierten Systemen verursacht (Hurni et al. 2008). 
- Pestizide sind immer noch wichtige Bestandteile von Pflanzenbausystemen. Gesundheitsschäden bei Landarbeitenden und Kleinbauern sowie Umweltschäden treten immer noch häufig auf (ILO 2000), obwohl sie durch geeignete Strategien reduziert werden könnten (World Bank 2007).

- Der Klimawandel wird weitreichende Folgen für die landwirtschaftliche Produktion haben. 13,5 Prozent der globalen Emissionen von Treibhausgasen, hauptsächlich $\mathrm{CH}_{4}$ und $\mathrm{N}_{2} \mathrm{O}$, stammen aus der Landwirtschaft (gemessen in $\mathrm{CO}_{2}$-Äquivalenten, siehe IPCC 2007, S. 36), was sie zu einer der bedeutendsten Quellen macht. Die Forstwirtschaft trägt weitere 17,4 Prozent bei (IPCC 2007, S. 36), vor allem aufgrund der Waldrodungen zur Gewinnung von Flächen für Land- und Viehwirtschaft. Die Erwärmung, mehr Niederschläge und der höhere $\mathrm{CO}_{2}$-Gehalt der Atmosphäre können positive Einflüsse auf die Produktion in den gemäßigten Zonen haben - jedoch nur, wenn sie moderat ausfallen. Extremereignisse wie Überschwemmungen und Dürren werden in vielen Gebieten zunehmen und sich zu den bereits bestehenden Herausforderungen addieren. Verschärfend kommt hinzu, dass gerade die am stärksten betroffenen Regionen (etwa semi-aride Gebiete im südlichen Afrika oder in Zentralasien) die geringsten Reaktionskapazitäten haben (IPCC 2007, World Bank 2007, IAASTD 2008).

- Die steigende Nachfrage nach Biotreibstoffen und die Energiekrise (World Bank 2007) könnten die Landwirtschaftssysteme und -märkte umfassend verändern. Grundsätzlich sind nachwachsende Energieträger Massengüter, die große Produktionsflächen und Anlagen benötigen, was eine Verdrängung von Bäuerinnen und Bauern durch kapitalkräftige Investoren zur Folge haben kann (IAASTD 2008). Kleinproduzenten haben allenfalls als dezentrale Energieproduzenten einen Konkurrenzvorteil. Wie zurzeit breit diskutiert wird, ist die ökologische Effizienz (im Hinblick auf Energieverbrauch und Treibhausgasemission) der Energiegewinnung aus Agrarprodukten in vielen Fällen fraglich (Fargione et al. 2008, IAASTD 2008). Eine weitere Folge der Energiekrise ist die zusätzliche Verteuerung der intensivierten landwirtschaftlichen Produktion durch die hohen Energiepreise (FAO 2000b).

\section{Die beiden Studien im Vergleich}

\section{International Assessment of Agricultural Knowledge, Science and Technology for Development (IAASTD)}

„Noch nie war es wichtiger, landwirtschaftliches Wissen, Wissenschaft und Technologie zu produzieren und einzusetzen“ (IAASTD 2008b, S. 4). Das IAASTD hat sich hohe Ziele gesetzt, nämlich nicht weniger als eine Neuorientierung der Wissensgenerierung und -nutzung im landwirtschaftlichen Bereich. Möglichkeiten zur Armutsbekämpfung und zur Erreichung ökologischer Ziele sollten untersucht und bewertet werden.

Bereits 2002 initiierten die Welternährungsorganisation (FAO) und die Weltbank einen Multi-Stakeholder-Prozess mit dem Ziel, die Rolle von Wissen, Wissenschaft und Technologie in der Landwirtschaft aus verschiedenen Perspektiven zu bewerten und Vorschläge für eine Neuausrichtung zu machen. Für den partizipativen Prozess sind sieben internationale Organisationen ${ }^{1}$ und ihre Mitgliedstaaten verantwortlich. Je dreißig Vertreter(innen) von Regierungen und der Zivilgesellschaft bildeten ein leitendes Gremium. An der Durchführung waren über 400 Expert(inn)en ${ }^{2}$ aus der Wissenschaft, Nichtregierungsorganisationen und dem Privatsektor beteiligt. Das Projekt wurde nach geografischen Kriterien in Teilprojekte aufgeteilt; zum Ergebnis gehören ein globales und fünf sub-globale Assessments. Das Verfahren ließ unterschiedliche Paradigmen $\mathrm{zu}$, berücksichtigte sowohl formale Wissenssysteme wie auch lokales Wissen, und das Ergebnis sollte „politikrelevant, aber nicht Politik vorschreibend“ (IAASTD 2008b) sein. Der konzeptionelle Rahmen und die Treffen wurden im vorbereitenden Prozess festgesetzt und den Autor(inn)en vorgegeben. Eine wichtige Vorgabe war auch, dass alle Aussagen auf bereits publizierte Literatur abgestützt, also überprüfbar sein müssen. Die Zusammenfassung der Ergebnisse wurde im April 2008 von 57 Staaten angenommen, während drei (Australien, Kanada und USA) Vorbehalte äußerten. ${ }^{3}$

Die bisher vorliegenden Dokumente vermitteln den Eindruck, dass das IAASTD die Ziele nicht vollständig erreichen konnte. Der Bericht hat zwar wichtige neue Akzente gesetzt und die Notwendigkeit eines neuen Ansatzes unterstrichen, weckt aber auch den Eindruck eines Panoptikums von Herausforderungen, ohne dabei einen Konsens über konkrete Maßnahmen herbeiführen zu können (Stokstad 2008).

Das IAASTD betont, dass trotz Produktivitätserfolgen eine Neuausrichtung von Forschung und Politik notwendig sei: Die Agrarwissenschaft müsse lokale Wissenssysteme stärker einbeziehen und neben der Produktion ökologische und soziale Ziele besser berücksichtigen (integrative Herangehensweise). In jedem Falle seien höhere Investitionen in die landwirtschaftliche Forschung nötig. Damit landwirtschaftliche Wissenssysteme einen Beitrag zu nachhaltiger Entwicklung leisten können, müssten entsprechende Änderungen auch in der Agrarpolitik eingeleitet werden. Die Lösung der anstehenden Probleme lasse sich

1 Food and Agriculture Organization of the United Nations (FAO), Global Environment Facility (GEF), United Nations Development Programme (UNDP), United Nations Environment Programme (UNEP), United Nations Educational, Scientific and Cultural Organization (UNESCO), Weltbank, World Health Organization (WHO).

2 Die Autor(inn)en dieses Artikels waren in unterschiedlichen Rollen am IAASTD-Prozess beteiligt: Hans Hurni war coordinating lead author, Markus Giger und Brigitte Portner waren contributing authors von Kapitel 1 des Global Assessment: Context, Conceptual Framework and Sustainability Indicators. Urs Scheidegger war lead author von Kapitel 6 des Global Assessment: Options to Enhance the Impact of AKST on Development and Sustainability Goals. („AKST“ steht für „,agricultural knowledge, science and technology“.)

3 Bei Redaktionsschluss lagen nur der Synthesis Report und eine Zusammenfassung in definitiver Form vor. Die Publikation der Hauptberichte wird für Herbst 2008 erwartet. Die Entwürfe sind noch nicht zur Zitierung freigegeben. Wir zitieren deshalb, wo möglich, direkt die zugrundeliegende Fachliteratur. 
nicht beliebig externalisieren oder in die Zukunft verschieben (weitere Botschaften siehe Tabelle 2, S. 284).

Das IAASTD hat bereits jetzt international eine breite Diskussion ausgelöst, begünstigt durch die aktuelle Hungerkrise. Die Diskussion hat sich vor allem auf die Kontroverse um gentechnisch modifizierte Organismen (GMO) fokussiert, ein Thema unter vielen anderen, die behandelt wurden. Ausgelöst wurde dies durch den Rückzug von Industrievertreter(inne)n aus dem Prozess mit der Begründung, die Biotechnologie werde zu negativ bewertet. Die Zeitschrift Nature Biotechnology wirft dem IAASTD vor, GMO ihren Nutzen beinahe vollständig abzusprechen (Nature Biotechnology 2008). Auch Nature bewertet das Kapitel über Biotechnologie als unausgewogen und übervorsichtig, wies aber darauf hin, dass andere Berichte (UN Millennium Project 2005) ebenfalls die Biotechnologie nicht als erste Priorität im Kampf gegen den Hunger betrachten, und kritisiert daher den Rückzug der Industrie (Nature 2008). Nach Stokstad (2008, S. 1476) rezipiert der Synthesebericht des IAASTD nicht die positiven Bewertungen der Biotechnologie aus den Hauptberichten. Die Autor(inn)en entgegnen, ihre Argumentation beruhe auf dem konkreten Nutzen von GMO für Kleinbauern in den letzten zehn Jahren gemäß der gesamten zur Verfügung stehenden wissenschaftlichen Literatur (Heinemann 2008).

Von entwicklungspolitischen Nichtregierungsorganisationen wurde der Bericht mit seinen Botschaften sehr begrüßt.

\section{World Development Report 2008 - Agriculture for Development (WDR)}

Der Bericht der Weltbank will die Landwirtschaft wieder in das Zentrum der Entwicklungsagenda und des Kampfs gegen die Armut stellen. Es ist das erste Mal seit 1982, dass dieser jährlich erscheinende Bericht das Thema globale Landwirtschaft als Fokus wählt - ein erstaunlich langer Zeitraum, wenn man bedenkt, dass Armut immer noch mehrheitlich im ländlichen Raum anzutreffen ist (Ravallion et al. 2007). Der Bericht wurde unter der Leitung zweier verantwortlicher Autoren von einem relativ kleinen Expertenteam verfasst. Zahlreiche Grundlagenberichte wurden in Auftrag gegeben. Zwischen 2006 und 2007 traf sich das Team mit weiteren Expert(inn)en aus Wissenschaft, Politik, Industrie und Nichtregierungsorganisationen und führte eine zweiwöchige öffentliche Konsultation über das Internet durch.

Die Förderung der Landwirtschaft sei ein Schlüsselprozess in der Armutsbekämpfung und deshalb seien vermehrte Investitionen in diesem Bereich notwendig - so lautet die Hauptbotschaft des Berichts. Damit ist die Publikation ein Meilenstein in der Entwicklungsdiskussion.

Die Analyse ist ökonomisch geprägt, auch wenn auf soziale und ökologische Probleme eingegangen wird. Zugrunde liegt ein Konzept von „drei Welten der Landwirtschaft“ in Entwicklungsund Schwellenländern (landwirtschaftlich geprägte Länder, Länder im Wandel und urbanisierte Länder) ${ }^{4}$ (Tabelle 2). Dementsprechend formuliert der Bericht seine Botschaften. Das erlaubt grundsätzlich eine differenzierte Herangehensweise, impliziert aber auch eine quasi notwendige Entwicklung. So zielt der Be- richt darauf, die Armen zur Teilnahme am marktwirtschaftlichen System zu befähigen. Wege dazu sind eine verbesserte governance auf allen Ebenen, Marktintegration, institutioneller Wandel durch Kooperationen zwischen Privaten und Öffentlichkeit sowie staatliche Unterstützung, um diese Entwicklungen zu ermöglichen.

Entwicklungspolitische Nichtregierungsorganisationen äußerten zum Teil massive Kritik am WDR, wenngleich auch diese Stimmen den neuen Fokus auf Landwirtschaft begrüßen. Der Bericht propagiere einseitig liberale Rezepte, die sich schon in der Vergangenheit nicht bewährt hätten (Patel 2007, S. 15 ff.). Die Konflikte, die aufgrund bestehender Machtstrukturen zu erwarten seien, blieben weitgehend unbeachtet. Ein Beispiel dafür sei die prekäre Situation von Kleinbauern in Wertschöpfungsketten (Hein 2008, S. 6, 7). Sowohl bei der Ausgestaltung wie auch beim Tempo von Liberalisierungen werde häufig keine Rücksicht auf Kleinbauern genommen, und die Betonung der ökonomischen Effizienz vernachlässige Gleichheitsprinzipien und Geschlechterrollen (Oxfam 2007). Auch das Prinzip der Ernährungssouveränität werde nicht aufgenommen. In diesem Zusammenhang werden eine historische Perspektive und die selbstkritische Analyse früherer Anstrengungen der Weltbank und ihrer Folgen vermisst (Murphy und Santarius 2007, S. 7, 25). So werde die harsche interne Kritik an der Politik der Weltbank nicht erwähnt, die Förderung der Landwirtschaft in Afrika sei ungenügend, nur punktuell und teilweise widersprüchlich (IEG 2007, S. 71 f.). Ohne solche Reflexion sei es nicht möglich, neue Ansätze zu entwickeln. Außerdem habe der WDR die Herausforderungen des Klimawandels und der Überwindung des Erdölzeitalters nicht ausreichend in die Analysen einbezogen und gehe deshalb am Kern der Sache vorbei (Murphy und Santarius 2007, S. 27).

\section{Übereinstimmungen}

Angesichts der Komplexität des Themas ist es nicht erstaunlich, dass beide Berichte mit einer großen Anzahl von Strategien und Strategieelementen aufwarten. Gemeinsam ist den Berichten die Schlussfolgerung, dass Landwirtschaft zentral für die Armutsbekämpfung ist. Der WDR (2008, S. 4) wartet dazu mit Daten auf: Die Landwirtschaft erweist sich für die Armutsbekämpfung als mindestens doppelt so effektiv wie andere Sektoren. Ein um ein Prozent steigendes Bruttosozialprodukt im Landwirtschaftssektor bringt zwei- bis dreieinhalbmal so viel wie das entsprechende Wachstum in anderen Sektoren. Vier Fünftel des beobachteten Rückgangs ländlicher Armut sind die Folge besserer Lebensbedingungen in ländlichen Gegenden, nicht die Folge von Emigration in urbane Gebiete. Ferner ist der Anteil der Armen an der Bevölkerung in marginalen Gebieten zwar höher, die größte Anzahl der Armen (61 Prozent) findet sich aber in relativen Gunstregionen in Asien und Lateinamerika. Die Mehrheit der Armen

\footnotetext{
4 „Agriculture-based countries, transforming countries, urbanized countries“ (World Bank 2007, S. 4). Gemäß dem WDR (2008, S. 4) können aber auch innerhalb eines Landes verschiedene Entwicklungsstadien vorkommen.
} 
lebt folglich in gut erreichbaren Regionen mit agrarökologisch guten Voraussetzungen, und Investitionen in die Landwirtschaft könnten effizient und mit Rücksicht auf die arme Bevölkerung gestaltet werden.

Damit dies erreicht werden kann, verlangen beide Berichte mehr Forschungsinvestitionen. Im IAASTD finden sich Daten zu Metaanalysen von Kosten und Nutzen der Agrarwissenschaft, die den Investitionen in die landwirtschaftliche Forschung hohe Renditen zuschreiben. So wurde der Nutzen von nur drei Innovationen der CGIAR-Agrarforschungszentren (Consultative Group on International Agricultural Research), nämlich Hochertragssorten von Reis und Weizen sowie biologische Bekämpfung der Maniokschmierlaus, als doppelt bis achtmal so hoch geschätzt wie die sieben Milliarden US-Dollar, die in diese Zentren seit deren Gründung investiert wurden (Raitzer 2003, S.1). Das IAASTD weist aber auch auf die methodischen Schwierigkeiten solcher Studien (zum Beispiel wird die Verteilung von Kosten und Nutzen nicht erfasst) und die Vernachlässigung von zum Teil bedeutenden externen Kosten und Nutzen hin.

Sowohl das IAASTD wie auch der WDR schreiben Staat und Politik einen hohen Stellenwert zu und betonen deren Rolle für die Bereitstellung öffentlicher Güter. Im Gegensatz zum IAASTD sieht der WDR den Staat aber lediglich als befähigend und regulierend, nicht als unterstützend und strukturfördernd. Dementsprechend muss sich der WDR für die internationale Ebene vorwerfen lassen, dass er eher Wege zeigt, wie sich die Armen in das nicht infrage gestellte globale Marktregime integrieren können, als zu fragen, welche Strukturen und Praktiken ihnen Priorität einräumen könnten (Murphy und Santarius 2007, S. 28).

\section{Unterschiede}

Trotz der grundsätzlich positiven Einschätzung der Rolle der Forschung lautet die Botschaft des IAASTD, dass Wissenschaft und Technologie in der Landwirtschaft neue Wege gehen müssen, um sich den Herausforderungen der Zukunft stellen zu können. Der Biotechnologie wird zwar ein großer potenzieller Beitrag zugeschrieben, aber ihr direkter Nutzen für bäuerliche Familien und Konsument(inn)en wird kontrovers beurteilt. Aus diesem Grund setzt der Weltagrarrat eher auf neue Formen von Forschung und governance, die stärker auf die Bedürfnisse der ressourcenarmen Kleinbetriebe und auf Umweltziele ausgerichtet werden sollen. Dies steht in einem klaren Gegensatz zum WDR, der zwar auch die Vorteile von Partnerschaften hervorhebt, der Biotechnologie aber einen eindeutigen Nutzen auch für Kleinbetriebe beimisst.

TABELLE 2: Gegenüberstellung der Studien zur globalen Landwirtschaft: International Assessment of Agricultural Knowledge, Science and Technology for Development (IAASTD 2008 a, 2008b) und World Development Report 2008 - Agriculture for Development (WDR; World Bank 2007).

\begin{tabular}{|c|c|c|}
\hline & IAASTD-Berichte & WDR - Agriculture for Development \\
\hline $\begin{array}{l}\text { Analysengebiet, } \\
\text { Gliederung }\end{array}$ & global und regional (einschließlich Norden) & $\begin{array}{l}\text { drei „Welten“ der Landwirtschaft in Entwicklungs- und } \\
\text { Schwellenländern: } \\
\text { - landwirtschaftlich geprägte Länder } \\
\text { - Länder im Wandel } \\
\text { " urbanisierte Länder }\end{array}$ \\
\hline Abgrenzung & $\begin{array}{l}\text { Landwirtschaft im weitesten Sinne (einschließlich Forstwirt- } \\
\text { schaft und Fischerei) }\end{array}$ & $\begin{array}{l}\text { Pflanzenbau, Viehhaltung, Agroforstwirtschaft und Aquakultur, } \\
\text { aber nicht Forstwirtschaft und Fischerei }\end{array}$ \\
\hline Fokus & $\begin{array}{l}\text { landwirtschaftliches Wissen, Wissenschaft und Technologie } \\
\text { für Entwicklung im weiteren Sinne }\end{array}$ & Beitrag der Landwirtschaft zur ökonomischen Entwicklung \\
\hline Botschaften & $\begin{array}{l}\text { - Metaanalysen von Kosten-Nutzen-Rechnungen zeigen einen } \\
\text { - In landwirtschaftliche Forschung muss mehr investiert werden. } \\
\text { - Der einseitige Fokus auf Ertrag hat zum Teil negative Konse- } \\
\text { quenzen für die Umwelt gebracht. } \\
\text { - Technologien (als Lösungsansätze) werden diskutiert. } \\
\text { - Der Nutzen gentechnisch modifizierter Organismen ist } \\
\text { umstritten. } \\
\text { - Kleinbauern und lokale Wissenssysteme müssen einbezogen } \\
\text { werden. } \\
\text { - Landwirtschaft ist multifunktional. } \\
\text { - Sozio-ökonomische Ungleichheiten und Geschlechterrollen } \\
\text { sind Anliegen. } \\
\text { - Überernährung und ungesunde Konsummuster sind } \\
\text { problematisch. } \\
\text { - Frühzeitige Öffnung der Märkte birgt Risiken. } \\
\text { - Exportorientierung nützt, hat aber auch negative Folgen. }\end{array}$ & $\begin{array}{l}\text { - Hohe soziale und wirtschaftliche Heterogenität der Landwirtschaft } \\
\text { verlangt differenzierte Politiken: } \\
\text { — landwirtschaftlich geprägte Länder: Produktivitätssteigerung der } \\
\text { Kleinbauern; } \\
\text { — Länder im Wandel: Verschiebung zu hochwertigen Produkten, } \\
\text { Stärkung der nicht landwirtschaftlichen Sektoren und Unter- } \\
\text { stützung zum Austritt aus der landwirtschaftlichen Beschäftigung; } \\
\text { urbanisierte Länder: Integration der Kleinbauern in Marktketten, } \\
\text { bessere Anstellungsbedingungen in Landwirtschaft und } \\
\text { Agrobusiness, Märkte für Umweltdienstleistungen. } \\
\text { Mehr Investitionen in Wissenschaft und Technologie sind nötig, } \\
\text { vor allem in die Biotechnologie (einschließlich der Entwicklung } \\
\text { gentechnisch modifizierter Organismen). Diese Investitionen } \\
\text { sollen den Armen nützen. } \\
\text { Diversifizierung, hochwertige Produkte und verbesserter } \\
\text { Marktzugang bieten neue Chancen. } \\
\text { Lokale, nationale und globale governance muss gestärkt werden. }\end{array}$ \\
\hline Prozess & $\begin{array}{l}\text { - Das IAASTD ist ein partizipativer Prozess mit über } 400 \text { Auto- } \\
\text { r(inn)en aus Entwicklungs-, Schwellen- und Industrieländern. } \\
\text { - Viele Teilnehmende bemängeln die undurchsichtige } \\
\text { Steuerung des Prozesses durch das IAASTD-Sekretariat. } \\
\text { - In den zwei Jahren ziehen sich viele Expert(inn)en zurück. }\end{array}$ & $\begin{array}{l}\text { - Ausgewählte Autor(inn)en mit klarem Auftrag verfassen den Bericht. } \\
\text { (Nicht publizierte) Hintergrundpapiere von Expert(inn)en werden } \\
\text { einbezogen. } \\
\text { - Stakeholder werden konsultiert. }\end{array}$ \\
\hline
\end{tabular}


Ein weiterer Unterschied zwischen den Berichten betrifft die Liberalisierung der Agrarmärkte. Der WDR vertritt eine Strategie der weiteren Liberalisierung und Stärkung der Märkte und fordert den Abschluss der Doha-Runde. Weil der WDR dennoch davon ausgeht, dass die Ärmsten sowie landwirtschaftlich dominierte Staaten eher benachteiligt sein werden, fordert er, dass bestimmte Bedingungen zum Schutz potenzieller Verlierer erfüllt werden müssen. Für den WDR scheinen aber grundsätzlich die Vorteile zu überwiegen, während das IAASTD für ein vorsichtiges Vorgehen plädiert und ausdrücklich vor zu schneller Öffnung warnt. Entsprechend fordert es besonderen Schutz für Kleinproduzenten und für die am wenigsten entwickelten Länder in der Doha-Runde sowie spezielle Unterstützung für Produkte aus kleinbäuerlichen Produktionssystemen.

Die größte Chance sieht der WDR für Bauernbetriebe, die in agrarökologischen Gunstregionen hochwertige Produkte für nationale und internationale Märkte herstellen (World Bank 2007, S. 58 ff.). Diese dynamischen Akteure seien in der Lage, relativ effizient Arbeitsplätze zu schaffen und Mehreinkommen in die ländlichen Gebiete zu bringen. Kritiker(innen) werfen dem Bericht jedoch eine zu optimistische Sicht vor. Beispielsweise werde ausgeblendet, dass die Arbeit meist von Frauen ausgeübt werde, die oft nur schlecht bezahlt und temporär angestellt würden, um die Kosten niedrig zu halten (Oxfam 2007). Die Monopolisierung der Wertschöpfungsketten durch große internationale Konzerne habe die Marktstellung der Kleinbetriebe geschwächt und deren Margen im Rohstoffhandel zugunsten mächtiger Marktteilnehmer schrumpfen lassen (Morisset 1997). Das IAASTD betont im Gegensatz zum WDR diese Probleme und diskutiert Optionen für ein Gegensteuern, beispielsweise die Stärkung von Bauernorganisationen oder die Schaffung regionaler Handelsbündnisse.

\section{Ausblick}

Was sind also die Aussichten für die globale Landwirtschaft? Es ist zu hoffen, dass sich die Erkenntnis des IAASTD durchsetzt, dass der Landwirtschaft, besonders kleinbäuerlichen Betrieben, aus sozialer, ökologischer und ökonomischer Sicht eine wichtige Rolle für die weitere globale Entwicklung zukommt. Die Landwirtschaft ist - so beide Berichte - für die Armutsbekämpfung, aber auch für die Bewältigung der Folgen des Klimawandels zentral. Ebenso muss die inputintensive großflächige Landwirtschaft ihren Beitrag leisten, einerseits zur Ernährungssicherung, aber auch durch nachhaltige Gestaltung und einen sorgsamen Umgang mit Ressourcen.

Ein wichtiger Grundstein für eine neue Landwirtschaftspolitik ist die Betonung der Multifunktionalität der Landwirtschaft: Von der Landwirtschaft und ihren Produkten und Dienstleistungen profitieren Gesellschaft, Ökonomie und Ökologie. Darauf sollte aufgebaut werden - ebenso wie auf der Erkenntnis, dass lokales, kontextspezifisches Wissen notwendig ist, um mit Hilfe von neuen Formen der Wissensproduktion und Innovation die Her-

\section{Die Landwirtschaft ist zentral für die Armutsbekämpfung sowie für die Bewältigung der Folgen des Klimawandels.}

ausforderungen der Zukunft anzugehen. Um solche Formen der Wissensproduktion zu ermöglichen, sind neben innovativen Denkansätzen auch Finanzierungsmechanismen erforderlich.

Auch der WDR bietet mit seinem analytischen Ansatz wichtige Einsichten, wie etwa, dass die Mehrheit der Armen in agrarökologischen Gunstregionen lebt und somit eine Verbesserung ihrer Lebensumstände und der landwirtschaftlichen Produktion durch relativ geringe Investitionen erreicht werden könnte. Davon würde die ganze Gesellschaft profitieren. Allerdings sollte es im Sinne der Nachhaltigkeit nicht darum gehen, im Schnellverfahren Vorgehensweisen festzuschreiben, sondern darum, einen breiten Aushandlungsprozess, an dem auch die direkt Betroffenen teilnehmen können, zu führen und nach Lösungen $\mathrm{zu}$ suchen. Dazu hat insbesondere das IAASTD einen wichtigen Beitrag geleistet.

Gerade strittige Themenbereiche wie die Biotechnologie sollten in einem gesellschaftlichen Diskurs geklärt und nicht allein den wirtschaftlichen Interessen überlassen werden, die sich in solchen Entscheidungen oft durchsetzen. Das IAASTD ist, trotz einiger Schwächen, ein erster solcher Versuch eines breit abgestützten Diskussionsprozesses, der über Expertenkreise hinausgeht. Es ist dieses Element, das das IAASTD hauptsächlich vom WDR unterscheidet und ihm einen besonderen Stellenwert gibt.

\section{Literatur}

Fan, S., B. Yu, A. Saurkar. 2008. Public spending in developing countries: Trends, determination, and impact. In: Public expenditures, growth, and poverty - Lessons from developing countries. Herausgegeben von S. Fan. Baltimore, MD: John Hopkins University Press. 20-55.

FAO (Food and Agriculture Organization of the United Nations). $2000 \mathrm{a}$. The state of food and agriculture 2000: Lessons from the past 50 years. Rom: FAO.

FAO. 2000 b. The energy and agriculture nexus. Rom: FAO.

FAO. 2005. The state of food and agriculture 2005: Agricultural trade and poverty: Can trade work for the poor? Rom: FAO.

FAO. 2006. The state of food and agriculture 2006: Food aid for food security? Rom: FAO

FAOSTAT. 2008. Statistical database of the FAO. Rom: FAO.

Fargione, J., J. Hill, D. Tilman, S. Polasky, P. Hawthorne. 2008. Land clearing and the biofuel carbon debt. Science 319/5867: 1235-1238.

Hein, W. 2008. Weltentwicklungsbericht 2008: Ein neues Konzept für die Landwirtschaft im Süden? GIGA Focus 1. Hamburg: German Institute of Global and Area Studies.

Heinemann, J. 2008. Off the rails or on the mark? Correspondence to the editor. Nature Biotechnology 26/5: 499-500.

Hurni, H., U. Christ, J. A. Lys, D. Maselli, U. Wiesmann. 2001. The challenges in enhancing research capacity. In: Enhancing research capacity in developing and transition countries: Experience, discussions, strategies and tools for building research capacity and strengthening institutions in view of promoting research for sustainable development. Herausgegeben von KFPE (Kommission für 
Forschungspartnerschaften mit Entwicklungsländern/Commission for Research Partnerships with Developing Countries). Bern: Geographica Bernensia. 50-58.

Hurni, H., K. Herweg, B. Portner, H. P. Liniger. 2008. Soil erosion and conservation in global agriculture. In: Land use and soil resources. Herausgegeben von A. K. Braimoh, P. L. G. Vlek. Heidelberg: Springer. 41-71.

IAASTD (International Assessment of Agricultural Knowledge, Science and Technology for Development). 2008a. IAASTD - Executive summary of the synthesis report. www.agassessment.org/docs/SR_Exec_Sum_210408_ Final.pdf (abgerufen 30.04.2008).

IAASTD. 2008b. IAASTD - Global summary for decision makers. www.agassessment.org/docs/Global_SDM_210408_FINAL.pdf (abgerufen 30.04.2008).

IEG (World Bank Independent Evaluation Group). 2007. World Bank assistance to agriculture in sub-Saharan Africa - An IEG review. Washington, D. C.: International Bank for Reconstruction and Development, World Bank.

ILO (International Labour Organization). 2000. Safety and health in agriculture. International Labour Conference, $88^{\text {th }}$ Session, Geneva. May 30 - June 15, 2000. Report VI/1. Genf: ILO.

IPPC (Intergovernmental Panel on Climate Change). 2007. Climate change 2007: Synthesis report. Genf: IPCC.

MA (Millennium Ecosystem Assessment). 2005. Our human planetSummary for decision makers. Washington, D. C.: Island Press.

Mazoyer, M. 2001. Protecting small farmers and the rural poor in the context of globalization. Rom: Food and Agriculture Organization of the United Nations (FAO).

Meinzen-Dick, R., M. Adato, L. Haddad, P. Hazell. 2004. Science and povertyAn interdisciplinary assessment of the impact of agricultural research. Washington, D. C.: International Food Policy Research Institute.

Morisset, D. 1997. Unfair trade: The increasing gap between world and domestic prices in commodity markets during the past 25 years. World Bank Economic Review 12/3: 503-526.

Murphy, S., T. Santarius. 2007. The World Bank's WDR 2008: Agriculture for development. Response from a slow trade - sound farming perspective. Ecofair Trade Dialogue Discussion Paper 10. Aachen/Berlin: Misereor/ Heinrich-Böll-Stiftung.

Nature. 2008. Deserting the hungry? Editorial. Nature 451: 223-224.

Nature Biotechnology. 2008. Off the rails. Editorial. Nature Biotechnology 26/3:247

Oxfam. 2007. What agenda now for agriculture? A response to the World Development Report 2008. Oxfam Briefing Note, October 19, 2007. www.oxfam.org/en/files/bn_what_agenda_for_agriculture_WDR_ 0710.pdf (abgerufen 19.02.2008).

Pardey, P. G., N. Beintema. 2001. Slow magic - Agricultural RaD a century after Mendel. Washington, D. C.: International Food Policy Research Institute.

Patel, R. 2007. The World Bank and agriculture - A critical review of the World Bank's World Development Report 2008. Discussion Paper. Johannesburg: ActionAid.

Raitzer, D. A. 2003. Benefit-cost meta-analysis of investment in the international agricultural research centres of the CGIAR. Prepared on behalf of the CGIAR Standing Panel on Impact Assessment. Rom: Science Council Secretariat, Food and Agriculture Organization of the United Nations (FAO).

Ravallion, M., S. Chen, P. Sangraula. 2007. New evidence on the urbanization of global poverty. World Bank Policy Research Paper 4199. Washington, D. C.: World Bank.

Stokstad, E. 2008. Dueling visions for a hungry world. Science 319/5869: 1474-1476.

Tilman, D., K. G. Cassman, P. A. Matson, R. Naylor, S. Polasky. 2002. Agricultural sustainability and intensive production practices. Nature 418: 671-677.

UN (United Nations). 2006. The millennium development goals report 2006. New York, NY: UN Department of Economic and Social Affairs.

UN. 2007. The millennium development goals report 2007. New York, NY: UN Department of Economic and Social Affairs.

UN (United Nations) Millennium Project. 2005. Halving hunger: It can be done. Summary version of the report of the Task Force on Hunger. New York, NY: The Earth Institute, Columbia University. von Braun, J. 2005. Small-scale farmers in liberalised trade environment. In: Small-scale farmers in liberalised trade environment. Proceedings of the Seminar October 2004, Haikko, Finland. Herausgegeben von T. Huvio, J. Kola, T. Lundström. Helsinki: Department of Economics and Management, University of Helsinki. 21-52.

Wood, S., K. Sebastian, S. J. Scherr. 2000. Pilot analysis of global ecosystems Agroecosystems. Washington, D. C.: International Food Policy Research Institute, World Resources Institute.

World Bank. 2007. World development report 2008 - Agriculture for development. Washington, D. C.: International Bank for Reconstruction and Development, World Bank.

Eingegangen am 6. Mai 2008; überarbeitete Fassung angenommen am 12. Juli 2008.

Markus Giger

Geboren 1957 in Bamenda, Kamerun. Studium der Agrarwissenschaften an der ETH Zürich, Nachdiplomstudium in Montpellier. Auslandsaufenthalte in Indonesien und Italien. Derzeit wissenschaftlicher Mitarbeiter am Zentrum für Entwicklung und Umwelt (Centre for Development and

Environment) des Geographischen Instituts der Universität Bern. Arbeitsgebiete: nachhaltige Ressourcennutzung und ländliche Entwicklung.

Hans Hurni

Geboren 1950 in Erlenbach im Simmental, Kanton Bern. Studium der Geografie. Aufenthalt in Äthiopien: 1975 bis 1977 Leiter eines Nationalparks, 1980 Dissertation in Klimatologie und Geomorphologie, 1981 bis 1987 Leiter eines nationalen Forschungsprogramms zur nachhaltigen Bodennutzung. 1991 Habilitation über Erosion und Schutz landwirtschaftlich genutzter Böden. Seit 1997 Titularprofessor an der philosophisch-naturwissenschaftlichen Fakultät der Universität Bern. Gegenwärtig Direktor des Nationalen Forschungsschwerpunkts Nord-Süd

(NCCR North-South) der Universität Bern sowie Leiter des Zentrums für Entwicklung und Umwelt (Centre for Development and Environment) des Geographischen Instituts der Universität Bern.

\section{Brigitte Portner}

Geboren 1979 in Oberdiessbach, Kanton Bern. Studium der Geografie, Ethnologie und Geologie an der Universität Bern. Wissenschaftliche Mitarbeiterin am Zentrum für Entwicklung und Umwelt (Centre for Development and Environment) des Geographischen Instituts der Universität Bern.

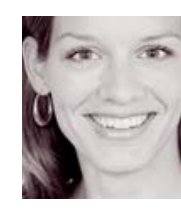

Urs Scheidegger

Geboren 1954 in Bern. Studium der und

Dissertation in Agrarwissenschaften an der ETH Zürich. Mitarbeiter internationaler Agrarforschungszentren in Lateinamerika und Afrika. Gegenwärtig Professor für tropische Pflanzenproduktion an der Schweizerischen Hochschule für Landwirtschaft, Zollikofen. Arbeitsschwerpunkte: Biodiversität und Farming Systems Research. 